\title{
An endocrinopathy characterized by dysfunction of the pituitary-adrenal axis and alopecia universalis: supporting the entity of a triple $H$ syndrome
}

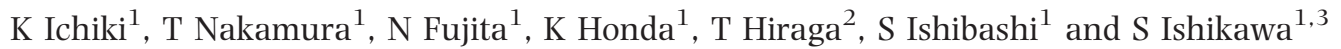 \\ ${ }^{1}$ Division of Endocrinology and Metabolism, Department of Medicine, ${ }^{2}$ Department of Dermatology, Jichi Medical School, Tochigi 329-0498, Japan and \\ ${ }^{3}$ Department of Medicine, Jichi Medical School Omiya Medical Center, Saitama 330-8503, Japan \\ (Correspondence should be addressed to S Ishikawa, Department of Medicine, Jichi Medical School Omiya Medical Center, 1-847 Amanuma, Saitama, \\ Saitama 330-8503, Japan; Email: saneiskw@jichi.ac.jp)
}

\begin{abstract}
We demonstrate the rare disorder of triple $\mathrm{H}$ syndrome in a 25-year-old man. He was pointed out as having short stature, at -5.9 S.D., and diagnosed as GH deficient at 6 years old. Approximately a year ago, he noticed systematic hair loss. He lost body weight by $7 \mathrm{~kg}$ during the last half year. He was admitted to Jichi Medical School Hospital because of unconsciousness. Physical findings showed disturbance of consciousness with Japan Coma Scale I-3. He had emaciation and alopecia universalis. Laboratory findings showed plasma glucose was as low as $1.11 \mathrm{mmol} / \mathrm{l}$. GH and ACTH deficiency with hypoadrenocorticism were clarified. His intelligence was in the low normal range with a WAIS IQ of 70, and anterograde amnesia was suggested in the presence of a little, but not significant, morphological change in the hippocampus on a magnetic resonance imaging scan. Replacement by a physiological dose of hydrocortisone normalized plasma glucose, and restored body weight and growth of hair during the 7 month therapeutic period. The present finding strongly supports a clinical entity of triple H syndrome, including ACTH deficiency, alopecia universalis and anterograde amnesia, and that there may be some variation of the triad among the subjects.
\end{abstract}

European Journal of Endocrinology 147 357-361

\section{Introduction}

Farooqi et al. (1) initially reported 'triple $\mathrm{H}$ syndrome' in two independent subjects, who developed the triad of isolated ACTH deficiency, alopecia and impairment of anterograde memory with radiological abnormalities in the hippocampus. The uncommon nature of the abnormalities may probably relate to an autoimmune mechanism, and the subjects were treated with a high dose of corticosteroids and azathioprine. According to this unusual triad of features, they suggested that the term triple $\mathrm{H}$ syndrome reflects the simultaneous dysfunction of the hippocampus, hair follicles, and the hypothalamic-pituitary-adrenal axis (1). In the present study we can add another subject with probable triple $\mathrm{H}$ syndrome, who had hypopituitarism including growth hormone (GH) and adrenocorticotropin (ACTH) deficiency, and alopecia universalis. Some variations are found among these three subjects as detailed below. The onset of ACTH deficiency and alopecia universalis seems simultaneous, but the hippocampal dysfunction is variable. It is not ruled out that there could be a deficiency of more than one pituitary hormone. In addition, physiological doses of hydrocortisone remarkably restored hair loss. The present study strongly supports the establishment of the clinical entity of triple $\mathrm{H}$ syndrome, and notes some variation among the subjects.

\section{Case report}

A 25-year-old man was admitted to Jichi Medical School Hospital because of unconsciousness. His birth had been normal with a height of $47 \mathrm{~cm}$ and a body weight of $2500 \mathrm{~g}$. At 6 years old he was pointed out as having short stature as his height was $85.5 \mathrm{~cm}$, deviated at -5.9 S.D. He was further examined at the hospital. Although the basal level of GH was within normal range, there was no response of $\mathrm{GH}$ secretion to several appropriate stimulations. His bone age was low, at $61 \%$ compared with his chronological age. He was diagnosed as isolated $\mathrm{GH}$ deficient. However, he had not taken medication because of undetermined reasons. At 10 years old specific treatment with s.c. administration of $\mathrm{GH}$ was started, and thereafter it had been continued for the last 9 years. When the 


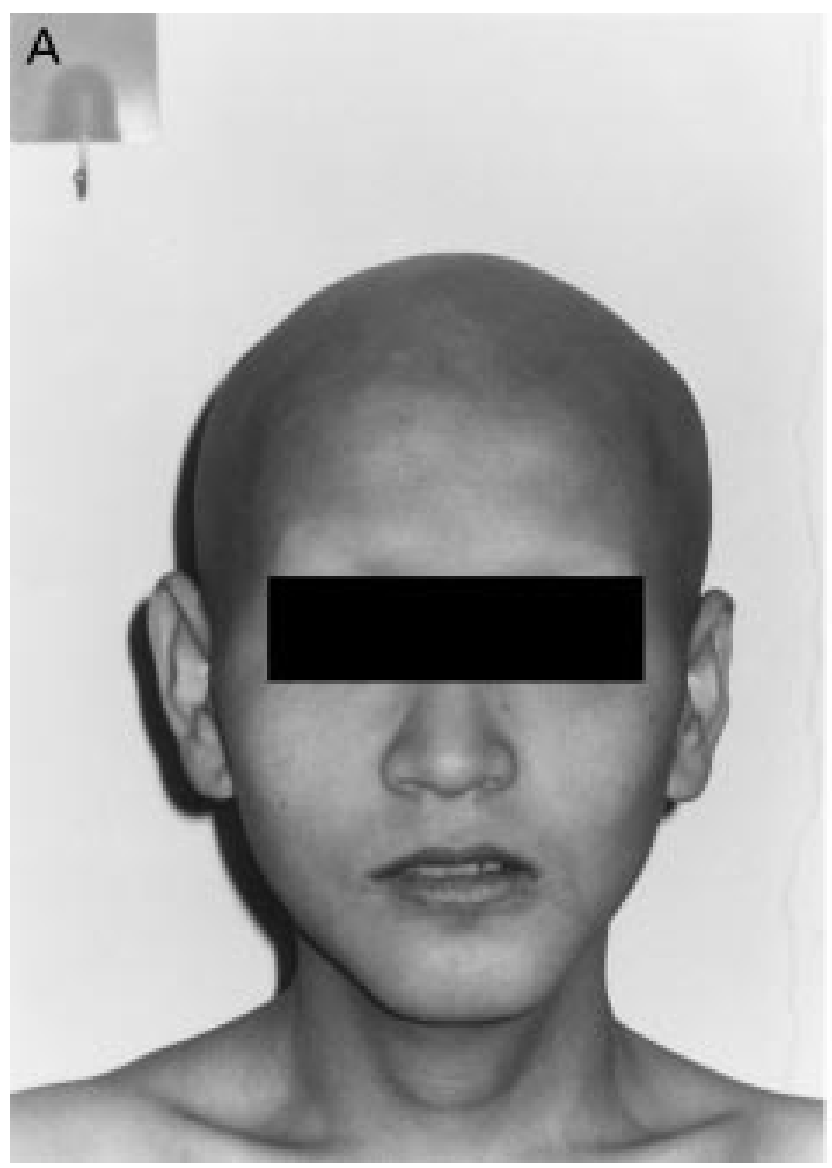

Figure 1 Picture of the patient.

treatment was withdrawn, his height had become $136.3 \mathrm{~cm}$ at 17 years old, which was still as low as - 6.0 S.D. Approximately a year ago he noticed his hair loss systematically, including hair, cilia, beard, axillary hair, pubic hair and leg hair. He consulted a physician about the hair loss, but no abnormality was found. Also he complained of appetite loss and generalized malaise. During the half year he lost body weight, from 35 to $28 \mathrm{~kg}$. On 13 January 2001, he ate only an orange and drank a can of juice. On January 14, he was admitted to Jichi Medical School Hospital because of unconsciousness. At the emergency room, the plasma glucose level was as low as $1.11 \mathrm{mmol} / \mathrm{l}$, and he became alert after an i.v. administration of glucose. Thereafter, he was hospitalized to examine further the pathogenesis of hypoglycemia.

Physical findings at hospitalization were height $143 \mathrm{~cm}(-4.8$ S.D.); body weight $27.8 \mathrm{~kg}$ with a body mass index of $13.6 \mathrm{~kg} / \mathrm{m}^{2}$; blood pressure $104 / 88 \mathrm{mmHg}$ without postural changes; pulse rate 78 beats/min with regular rhythm; and body temperature $35.4{ }^{\circ} \mathrm{C}$. His consciousness deteriorated by degree of the Japan Coma Scale I-3 (2). He had no dry skin and tongue. Hair, cilia, beard, axillary hair, pubic hair

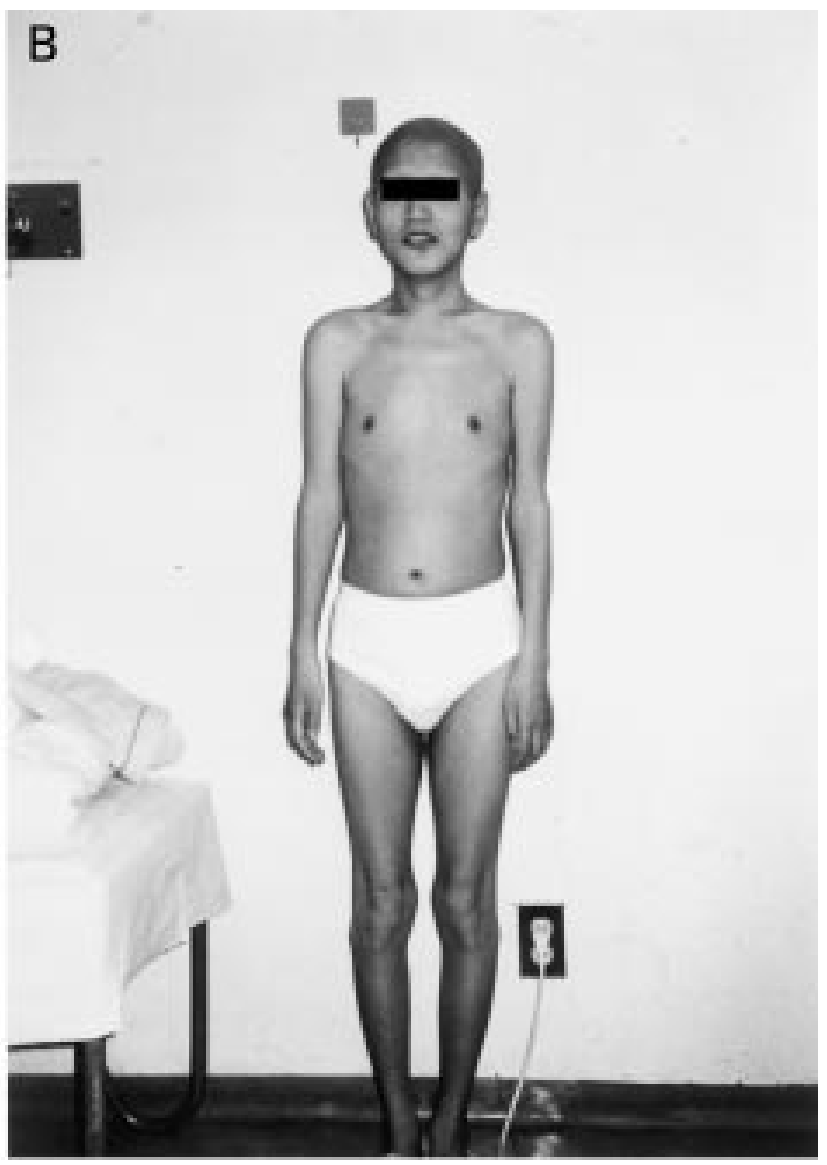

and leg hair were totally lost (Fig. 1). Neurological examination showed no abnormal findings.

Laboratory studies showed white blood cells were $9700 / \mathrm{mm}^{3}$; red blood cells $400 \times 10^{4} / \mathrm{mm}^{3}$; hemoglobin $11.9 \mathrm{~g} / \mathrm{dl}$; hematocrit $35.2 \%$; and platelets $27.5 \times 10^{4} / \mathrm{mm}^{3}$. Serum sodium level was $137 \mathrm{mmol} / \mathrm{l}$; potassium $3.6 \mathrm{mmol} / \mathrm{l}$; and chloride $102 \mathrm{mmol} / \mathrm{l}$. Blood urea nitrogen level was $4.64 \mathrm{mmol} / \mathrm{l}$; serum creatinine $51.3 \mu \mathrm{mol} / \mathrm{l}$; and serum uric acid $256.0 \mu \mathrm{mol} / \mathrm{l}$. Plasma glucose level was $1.11 \mathrm{mmol} / \mathrm{l}$ and hemoglobin $A_{1 \mathrm{c}} 4.3 \%$. Serum total cholesterol was $2.77 \mathrm{mmol} / \mathrm{l}$ and triglyceride $0.67 \mathrm{mmol} / \mathrm{l}$. A urinalysis showed no abnormalities. Basal levels of serum or plasma pituitary hormones were: $\mathrm{GH} 0.8 \mu \mathrm{g} / \mathrm{l}$; follicle-stimulating hormone (FSH) $11 \mathrm{IU} / \mathrm{l}$; luteinizing hormone (LH) $9 \mathrm{IU} / \mathrm{l}$; thyrotropin (TSH) $13.7 \mathrm{mU} / \mathrm{l}$; prolactin $16 \mu \mathrm{g} / \mathrm{l}$; and ACTH below $1 \mathrm{pmol} / \mathrm{l}$. Serum cortisol was below $27.6 \mathrm{nmol} / \mathrm{l}$; dehydroepiandrosterone (DHEA) $3.1 \mathrm{nmol} / \mathrm{l}$; and DHEA sulfate $0.28 \mu \mathrm{mol} / \mathrm{l}$. Serum free thyroxine and free triiodothyronine were 11.6 and $2.7 \mathrm{pmol} / \mathrm{l}$ respectively. Urinary excretions of 17-ketosteroids (17-KS) and 17-hydroxycorticosteroids (17-OHCS) were 2.1 and $1.7 \mathrm{mg} /$ day respectively. The stimulation tests for 
Table 1 The endocrinological data from various stimulation tests for pituitary hormones.

\begin{tabular}{lrr}
\hline & Basal level & Peak level \\
\hline CRH test & & \\
ACTH (pmol/l) & $<1.0$ & $<1.0$ \\
Cortisol (nmol/l) & $<27.6$ & $<27.6$ \\
Insulin tolerance test & & \\
Glucose (mmol/l) & 3.4 & 1.3 \\
ACTH (pmol/l) & $<1.0$ & $<1.0$ \\
Cortisol (nmol/l) & $<27.6$ & 0.1 \\
GH $(\mu \mathrm{g} / \mathrm{l})$ & 0.1 & 0.1 \\
GRH test & & \\
GH $(\mu \mathrm{g} / \mathrm{l})$ & 0.1 & 31.2 \\
TRH test & & 34.0 \\
TSH $(\mathrm{mU} / \mathrm{l})$ & 9.8 & 34.0 \\
Prolactin $(\mu \mathrm{g} / \mathrm{l})$ & 7.2 & 24.0 \\
LH-RH test & & \\
LH $(\mathrm{IU} / \mathrm{l})$ & 5.1 & \\
FSH $(\mathrm{IU} / \mathrm{l})$ & 13.0 & \\
\hline
\end{tabular}

pituitary hormones had been performed before the start of hydrocortisone replacement. Plasma ACTH and serum cortisol remained unchanged in response to either corticotropin-releasing hormone (CRH) or insulin-induced hypoglycemia (Table 1), but urinary excretions of 17-KS and 17-OHCS were markedly increased after the repeated i.m. administration of cortrosin-Z. Also, there was no alteration of serum GH in response to either GRH or insulin-induced hypoglycemia. There were normal responses of FSH, LH, TSH and prolactin to an appropriate stimulation of LHreleasing hormone (LH-RH) or thyrotropin-releasing hormone (TRH). A thyroid test, a microsome test, and tests for anti-thyroid peroxidase antibody, anti-thyroglobulin antibody, anti-intrinsic factor antibody, antiGAD antibody and anti-pituitary antibody were all negative.

A skin biopsy of the head demonstrated that hair follicles retained their original structure, but there was lymphocyte infiltration around hair follicles and around small vascular beds in subcutaneous and deep skin layers (Fig. 2).

A skull X-ray showed no deformity of the sella turcica. Brain magnetic resonance imaging (MRI) at coronal and sagittal projections showed mild empty sella with probably a mild atrophic change of the anterior pituitary gland. In a horizontal brain MRI, the right hippocampus seemed to be a little atrophic, a finding not suggesting its pathological significance.

Neuropsychological evaluation showed that intelligence was in the low normal range, as the estimated IQ score was 70 on the test of general intellectual ability (WAIS-R), i.e. scores for language ability and performance ability were 76 and 67 respectively. There might have been possible impairment in shortterm memory with word recognition memory, but not in long-term memory, using the Benton visual memory test (Table 2).

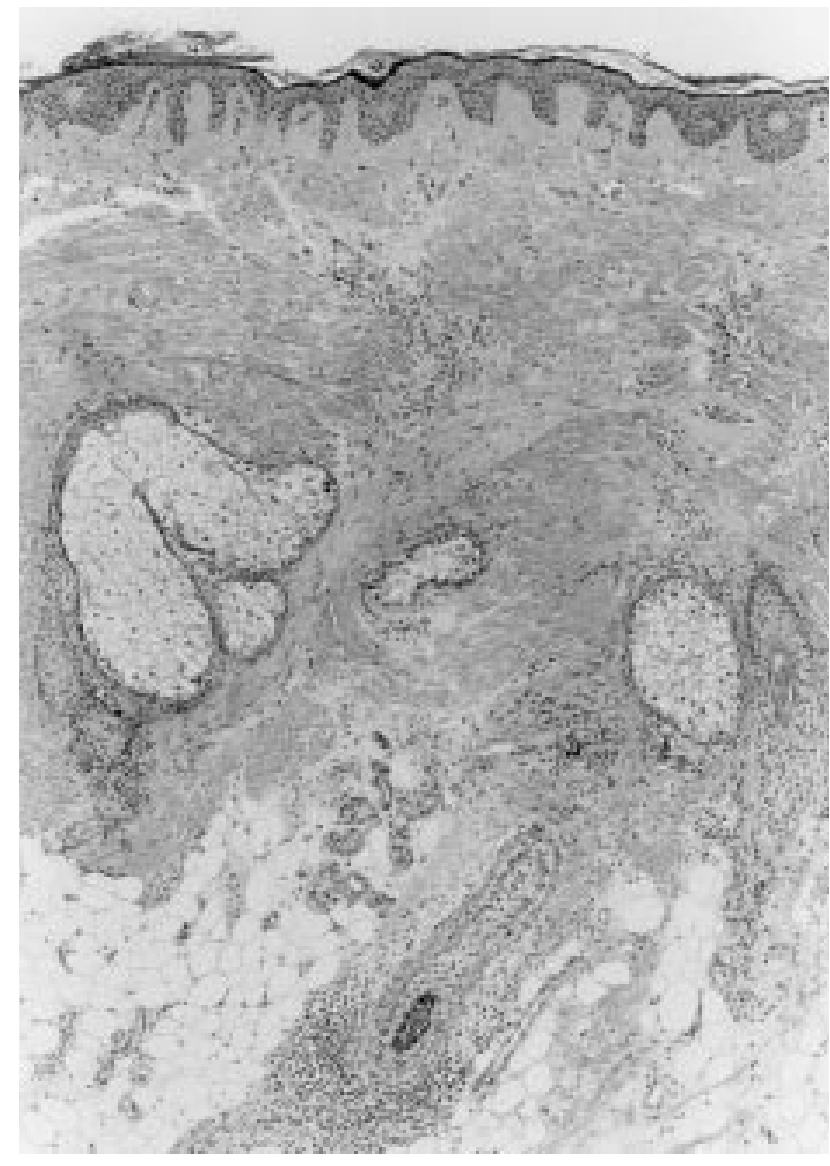

Figure 2 Microscopic finding of head skin. Hematoxylin-eosin staining. Magnification $\times 14$.

\section{Clinical course}

After completing the diagnosis of hypopituitarism (GH and ACTH deficiency), hydrocortisone replacement, at $20 \mathrm{mg}$ per day, was begun. After the start of the replacement therapy the patient became free from the symptoms of easy fatigability and disturbance of consciousness. Also, plasma glucose levels were maintained in the normal range and his body weight increased from 27.8 to $41.5 \mathrm{~kg}$ during the therapeutic period of 7 months. The disorder of his hair loss also disappeared, as hair, cilia, beard, axillary hair, pubic hair and leg hair reappeared by approximately $80-90 \%$ of the estimated normal condition, as shown in Fig. 3.

Table 2 Cognitive function tests in the patient.

\begin{tabular}{lc}
\hline Intelligence/WAIS-R & \\
Total IQ & 70 \\
Language IQ & 76 \\
Performance IQ & 67 \\
Memory/Benton visual retention test & 3 (estimated: 6*) \\
Accurate answers & 8 (estimated: 6*) \\
Inaccurate answers &
\end{tabular}

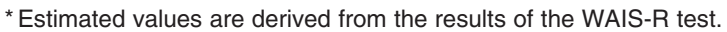



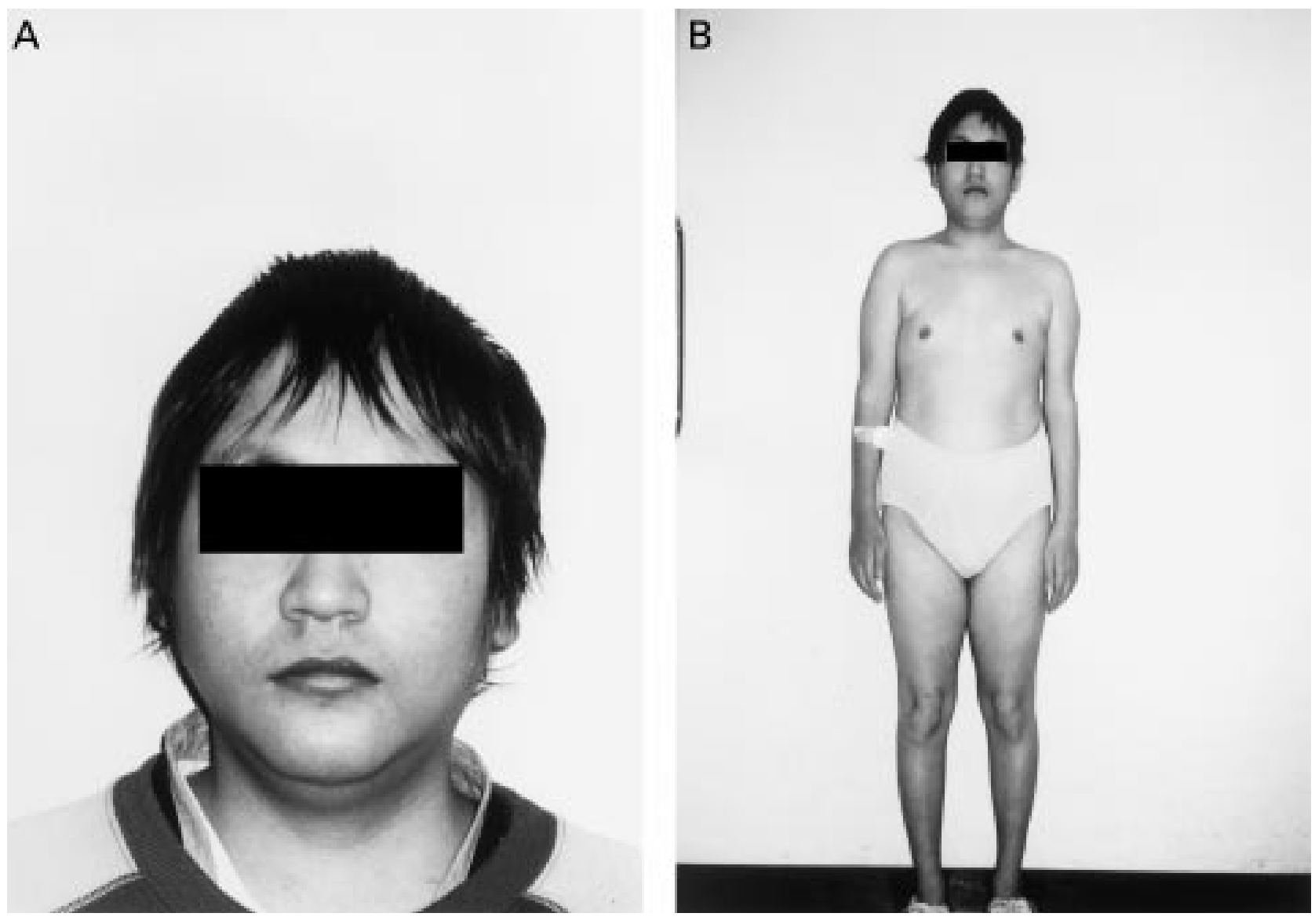

Figure 3 Picture of the patient 7 months after the start of hydrocortisone replacement.

\section{Discussion}

At the present episode the patient was suffering from secondary hypoadrenocorticism. He had lost appetite and body weight progressively and his plasma glucose level was as low as $1.11 \mathrm{mmol} / \mathrm{l}$, producing hypoglycemic coma at the emergency admission. However, hyponatremia was absent (3-5). Approximately a year prior to the present episode he had noticed systemic hair loss. Thus, alopecia universalis seemed likely to be closely associated with secondary adrenal insufficiency. We cannot comment about the progression of mental disturbance. Two clinical features were manifest, and anterograde amnesia might follow thereafter. From this point of view, the present patient should be diagnosed as having triple $\mathrm{H}$ syndrome, which was first reported by Farooqi et al. (1) with two independent subjects. Therefore, our patient is the third one at the present time.

Deficiency of ACTH and GH was evident in the present patient. The GH deficiency had existed for almost two decades and resulted in dwarfism. In the initial study of Farooqi et al. (1), isolated ACTH deficiency was only found as an endocrine disorder. We may suggest that the deficit of hormonal secretion shows more than one hormone in hypopituitarism associated with triple $\mathrm{H}$ syndrome. Morphological studies showed only mild empty sella, which was closely related to atrophic change of the pituitary gland itself (6), and there was no space-occupying lesion in the hypothalamus and pituitary gland. Alopecia universalis was associated with infiltration of lymphocytes in hair follicles in the skin biopsy of the head, raising speculation of an autoimmune mechanism $(7,8)$. Dysfunction of the hypothalamo-pituitary-adrenal axis as well as alopecia universalis may be linked to glandular autoimmunity $(9-11)$, although there was no positive antibody in the patient. We may have to consider the site of the lesion in the hypothalamo-pituitary axis in the present disorder. Since there are three different tissues or organs derived from the ectoderm, and systemic abnormality in hair follicles, hippocampus and hypothalamo-pituitary axis was suggested, a hypothalamic lesion seemed to be most probable $(12,13)$. This may be understandable if the disorders were based on autoimmunity. In addition, there was no change in ACTH in response to insulin-induced hypoglycemia, in which the stimulation should be mediated 
via the hypothalamus. Repeated stimulation with $\mathrm{CRH}$ might increase ACTH secretion, but we carried out the $\mathrm{CRH}$ test with only a single dose of $\mathrm{CRH}$. Taken together, hypothalamic dysfunction may have resulted in ACTH and GH deficiency in the present patient.

The initial study by Farooqi et al. (1) described the impairment of anterograde memory included as one of the triad in the triple $\mathrm{H}$ syndrome. Mild hippocampal atrophy was found in MRI scans $(14,15)$. However, we could not conclusively determine anterograde amnesia in the present patient, whose MRI scan did not clearly show morphological changes in the hippocampus. This discrepancy may suggest that the progression of each disturbance is different among the three disorders in the triple $\mathrm{H}$ syndrome. It is not ruled out that the anterograde amnesia will become manifest during the future follow-up observation period.

The onset of alopecia was detected by the patient approximately a year before the admission, and the progression of alopecia was very rapid, resulting in alopecia universalis (Fig. 1). Lymphocyte infiltration around hair follicles and small vascular beds was not inconsistent with autoimmune disorders. As shown in Fig. 3, his hair has been restored by about $80-90 \%$ of the estimated normal condition during the 7 month therapeutic period, although the hydrocortisone replacement was performed with $20 \mathrm{mg}$ per day, a dose in the physiological range. This finding also may suggest that alopecia universalis occurred related to ACTH deficiency, and that the mechanism of the pathogenic skin immunity was corrected by the replacement of physiological doses of hydrocortisone. Again, if we consider the site of the lesion causing ACTH and GH deficiency, disturbance of the anterior pituitary gland per se cannot be ruled out. From this point of view, anterograde amnesia might not be considered as part of the syndrome, because it was not evident in the present patient.

In conclusion, we have demonstrated a third case of triple $\mathrm{H}$ syndrome. ACTH deficiency and alopecia universalis seemed to develop simultaneously, but the progression of anterograde amnesia may be slow. The onset of the syndrome could be sporadic. Physiological doses of hydrocortisone corrected the patient's secondary hypoadrenocorticism, and also corrected his alopecia universalis. The present finding strongly supports a clinical entity of triple $\mathrm{H}$ syndrome, including dysfunction of the hypothalamo-pituitary-adrenal axis, hair follicles and hippocampus, and that there may be some variation of the triad among subjects. Further study will be necessary to establish the entity of triple $\mathrm{H}$ syndrome.

\section{References}

1 Farooqi IS, Jones MK, Evans M, Orahilly S \& Hodges JR. Triple H syndrome. A novel autoimmune endocrinopathy characterized by dysfunction of the hippocampus, hair follicle, and hypothalamic-pituitary-adrenal axis. Journal of Clinical Endocrinology and Metabolism $2000852644-2648$.

2 Takagi K, Aoki M, Ishii T, Nagashima Y \& Narita K. Japan coma scale as a grading of subarachnoid hemorrhage: a way to determine the scale. No Shinkei Geka 199826 509-515 (In Japanese).

3 Slessor A. Studies concerning the mechanism of water retention in Addison's disease and in hypopituitarism. Journal of Clinical Endocrinology 195111 700-723.

4 Ishikawa S, Fujisawa G, Tsuboi Y, Okada K, Kuzuya T \& Saito T. Role of antidiuretic hormone in hyponatremia in patients with isolated adrenocorticotropic hormone deficiency. Endocrinologia Japonica $199138325-330$.

5 Ishikawa S, Saito T, Fukagawa A, Higashiyama M, Nakamura T, Kusaka I et al. Close association of urinary excretion of aquaporin-2 with appropriate and inappropriate arginine vasopressindependent antidiuresis in hyponatremia in elderly subjects. Journal of Clinical Endocrinology and Metabolism 200186 1665-1671.

6 Ishikawa S, Furuse M, Saito T, Okada K \& Kuzuya T. Empty sella in control subjects and patients with hypopituitarism. Endocrinologia Japonica 198835 665-674.

7 Cecchi R, Giomi A, Tuci F, Bartoli L \& Seghieri G. Pityriasis rubra pilaris, lichen planus, alopecia universalis and vitiligo in a patient with chronic viral hepatitis C. Dermatology 1994188 239-240.

8 McDonagh AJ \& Messenger AG. The aetiology and pathogenesis of alopecia areata. Journal of Dermatological Science 19947 S125-S135.

9 Escobar-Morreale H, Serrano-Gotarredona J \& Varela C. Isolated adrenocorticotropic hormone deficiency due to probable lymphocytic hypophysitis in a man. Journal of Endocrinological Investigation $199417127-131$.

10 Crock PA. Cytosolic autoantigens in lymphocytic hypophysitis. Journal of Clinical Endocrinology and Metabolism $1998 \quad 83$ 609-618.

11 Nishiki M, Murakami Y, Ozawa Y \& Kato Y. Serum antibodies to human pituitary membrane antigens in patients with autoimmune lymphocytic hypophysitis and infundibuloneurohypophysitis. Clinical Endocrinology 200154 327-333.

12 Velardo A, Pantaleoni M, Zizzo G, Del-Rio G, Colletta F, Carani C et al. Isolated adrenocorticotropic hormone deficiency secondary to hypothalamic deficit of corticotropin-releasing hormone. Journal of Endocrinological Investigation 199215 53-57.

13 Nishihara E, Kimura H, Ishimaru T, Kiriyama T, Yokoyama N, Yamashita $\mathrm{S}$ et al. A case of adrenal insufficiency due to acquired hypothalamic CRH deficiency. Endocrine Journal $1997 \quad \mathbf{4 4}$ $121-126$.

14 Graham KS \& Hodges JR. Differentiating the role of the hippocampal complex and the neocortex in long-term memory storage: evidence from the study of semantic dementia and Alzheimer's disease. Neuropsychology 199711 77-89.

15 Kapur N \& Brooks PJ. Temporally-specific retrograde amnesia in two cases of discrete bilateral hippocampal pathology. Hippoсатриs 19999 247-254.

Received 13 March 2002

Accepted 29 May 2002 